\title{
ADVANCED PLANNING
}

\author{
A. Pienaar \\ iPlan Industrial Engineers, South Africa \\ abrepienaar@iplan.co.za
}

\begin{abstract}
"Advanced Planning" is often positioned, sometimes by vested interests, as the "latest version" of supply chain planning which "replaces" previous methods. The objective of this article is to clarify some of the concepts, present some historical perspectives, describe the essential elements and address the applicability of Advanced Planning. The key principle that is explored is that “Advanced Planning” represents an alternative planning methodology.

\section{OPSOMMING}

Gevorderde Beplanning word dikwels beskryf as die jongste metode waarvolgens leweringsnetwerke beplan behoort te word. Die indruk word geskep dat Gevorderde Beplanning vorige metodes vervang. Hierdie artikel verduidelik sommige van die relevante begrippe. 'n Historiese perspektief van die ontwikkeling van Gevorderde Beplanning word gelewer, die kernelemente word beskryf en die toepaslikhied van Gevorderde Beplanning bespreek. Die grondbeginsel van die artikel is dat Gevorderde Beplanning ' $n$ alternatiewe beplanningsmetodologie verteenwoordig.
\end{abstract}




\section{INTRODUCTION}

"Advanced Planning" is a term that is currently (at the end of 2003) used and often misused to describe almost any planning mechanism that is deemed to be better in some way than that which came before. This is especially true when one peruses the sales literature of software products that support activities in supply chain management and logistics.

On the other hand, there is a developing consensus by practitioners in the field that Advanced Planning does represent a significant breakthrough in the ability of organisations to achieve superior business results.

In the next few pages the origins of Advanced Planning are identified, the building blocks of Advanced Planning are discussed and some conclusions are drawn that may help practitioners consider the applicability of Advanced Planning.

\section{BUSINESS PROCESS APPROACH}

Advanced Planning is best understood in terms of the business process approach described in the industry-standard "SCOR"-model of the Supply Chain Council [10]. This model, shown graphically in figure 1, presents a business process as consisting of the methods, systems, data, procedures, performance measurements, roles and responsibilities used to plan and execute activities such as the buying, making and delivering of products and services.

In the context of the SCOR-model in figure 1 , the "Plan" activity may be performed using Advanced Planning or alternatively with "conventional" or "not-advanced" planning. This statement promptly leads to the question: "What is the difference?"

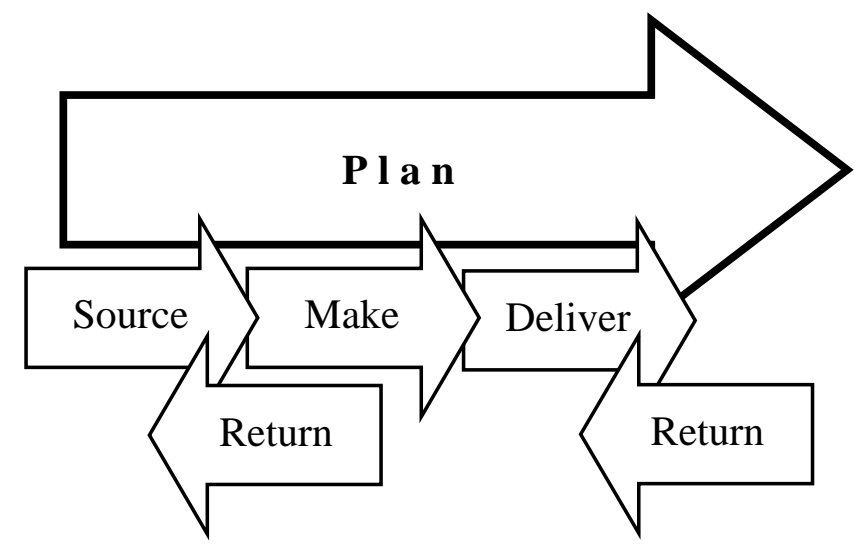

Figure 1: The SCOR model [10]

The short answer is that with Advanced Planning a computer system finds alternative feasible plans and selects the optimum, all in a single step and without human interaction whereas other ways of planning use a mixture of computer systems and human expertise in sequential steps to develop a feasible plan that may or may not be optimum.

The long answer is the content of this article.

\section{FEASIBLE AND OPTIMUM}

Consider by way of example a machine where several products have to be processed in a single work-shift. Machine set-up is significant and is dependent on the sequence in which the products are produced.

A planner scheduling this machine will evaluate alternative sequencing schemes. If the 
planner finds a sequence that allows all products to be completed within the work-shift, he or she will have found a feasible plan and may well stop the planning process at this point.

The planner may also continue to see if there are other feasible plans that call for different sequencing of products on the machine. Assume a number of such alternative, feasible plans are found after careful consideration of all possible sequencing alternatives. The planner now selects the plan that will result in the shortest overall production time on the machine. This is the optimum plan.

The essential concepts than can be extracted from this scheduling example are the following:

1. There may be many alternative ways to achieve the same objective. (In the example, the number of permutations of the sequence is calculated as $n !$.)

2. A feasible plan satisfies all constraints. (In the example this means a plan that will ensure all products are completed in a single shift.)

3. There may be no feasible plan, just one feasible plan or more than one feasible plan.

4. The optimum plan is that feasible plan which is deemed to be the best in terms of some criterion. (In the example this would be the shortest overall production time on the machine.)

\section{A COMPARISON OF METHODS}

The concepts above lead one to the following conclusions when considering different ways of planning.

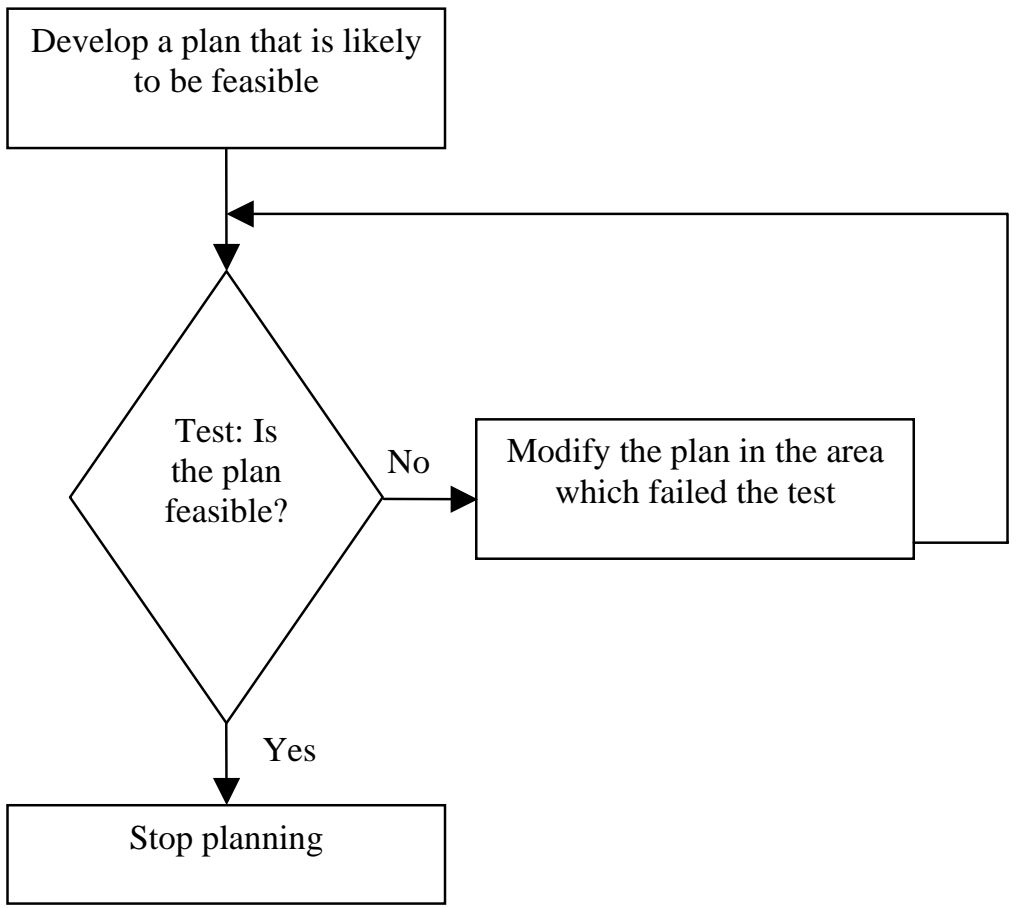

Figure 2: Developing a feasible plan
If the objective of the planning process is to develop a feasible plan, any feasible plan, the practical approach illustrated by the steps of the flow diagram in figure 2 are followed by many so-called "manual" systems, for example planning boards, gantt charts and graphic methods.

On the other hand, if the objective of the planning process is to find the optimum plan, if it exists, then one should first find all the possible feasible plans and then determine which of them represents the optimum. The flow diagram in figure 3 (following) shows the steps required to develop an optimum plan. 
Note that the description "optimum" automatically implies feasibility. If there are no feasible solutions to a problem, there can be no optimum.

Optimisation thus requires all of the following elements:

1. Consideration of alternative plans

2. Selection of the feasible, optimum plan.

3. The knowledge that no better plan exists.

\section{LINEAR PROGRAMMING}

The criterion that one uses to select the "optimum" plan requires quantification in order to perform the selection from amongst all the feasible alternatives. In the scheduling example above, the total time for the processing of all the products has to be calculated for each alternative plan. This quantification of a plan is known as the objective function.

The requirements that have to be satisfied - in the example above that all products must be processed in a single shift - are known as the constraint".

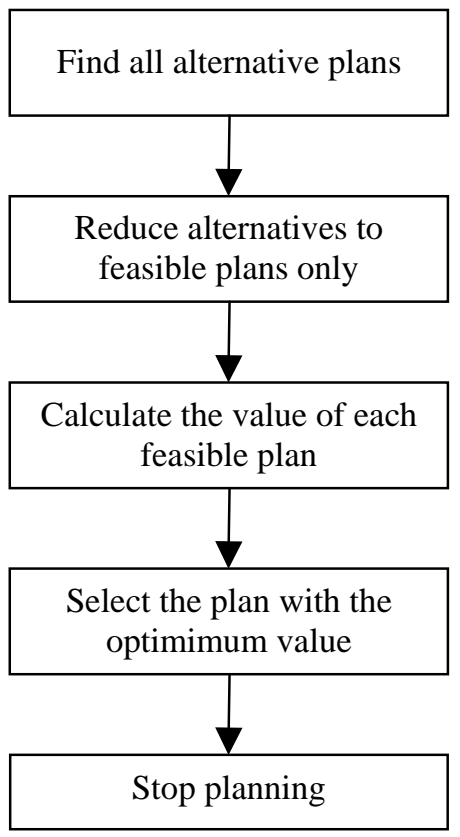

\section{Figure 3: Finding the optimum plan}

While the steps in developing an optimum plan are fairly straightforward - as can be seen in the linear sequence in figure 3 - the number of alternatives in all but the most trivial of problems one encounters in practice is usually so large that finding all alternatives, evaluating each in terms of the feasibility constraints, quantifying each feasible alternative in terms of the objective function and then selecting the optimum plan is just not practical.

In the 1940's, mathematicians developed a method called linear programming to solve the optimisation problem for a particular class of problems, those where the feasibility requirements and the objective function can be stated as first order mathematical equations. Essentially linear programming allows one to evaluate all alternative plans in a single step that is assured to unambiguously determine whether at least one feasible solution exist and if so to present the optimum feasible solution in terms of the objective function.

Using linear programming, the optimisation flow chart in figure 3 above can be reduced to the one in figure 4 .

In the decades after the 1940's similar methods were developed in the field that became known as operations research. Integer programming solves problems where the optimum plan may only have integer values in the result. Dynamic programming solves problems stated in multiple stages. Other operations research techniques address particular aspects of non-linear

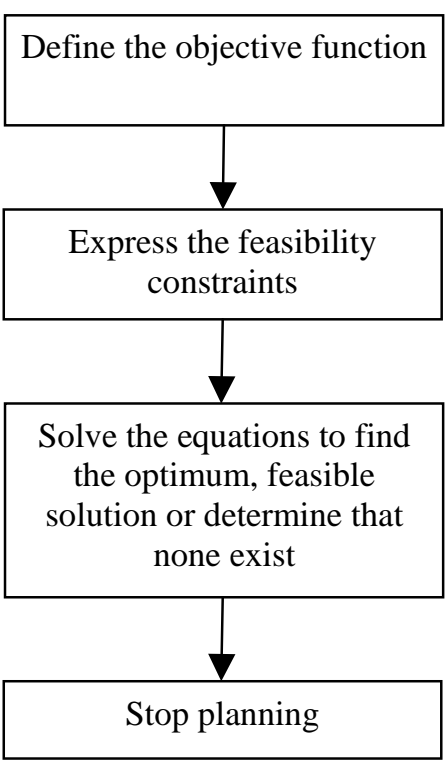

Figure 4: Optimisation with linear programming 
relationships. (The scheduling problem in the example above would probably be formulated as one of the latter and not as a linear programming model.)

As far as practical application goes, though, operations research remained a somewhat esoteric area of interest for most of the twentieth century. Practical applications tended to be restricted to very large and complex problems which were deemed to be worth the time and cost of people with the higher levels of mathematical training required for formulating and solving the mathematical equations.

\section{REQUIREMENTS PLANNING}

The business world went in a different direction.

In the late fifties Joseph Orlicky [6] developed a way to address a planning problem of particular significance to manufacturing companies - scheduling the supply of raw materials and components required for manufacturing. In order to ensure all material would be available when required for production, the typical approach at the time was to use order point techniques. Order point planning in practice meant that the company would have inventory of raw materials at all times; thus usually trading off the assurance of no material shortages for high levels of raw material and component inventory.

Orlicky's method, called "Requirements Planning”, synchronises the planning of all the dependent materials in such a way that the overall inventory level required to support the same production schedule is significantly lower than with order points. Moreover, Requirements Planning is based on a very simple four-question approach that is intuitively understood:

1. How many do we need and when do we need it?

2. How many do we have?

3. How many have we ordered and when will we get it?

4. How many and for when must we now plan?

For example, if we need 60 units of some raw material (question 1) and we have 20 in stock right now (question 2) with another 15 on order (question 3), then we should plan to get another 25 .

The only disadvantage of Orlicky's method was that a manufacturing business would require a vast number of cycles through this very simple four-step algorithm to plan its operations. This practical obstacle meant that for most of the sixties Requirements Planning remained no more than an interesting academic approach.

This changed immediately with the introduction of computers into manufacturing in the late sixties and early seventies. If we leave time-phasing out of the calculation for the moment (as in the numeric example above) Orlicky's

1. How many do we need and when do we need it?

2. How many do we have?

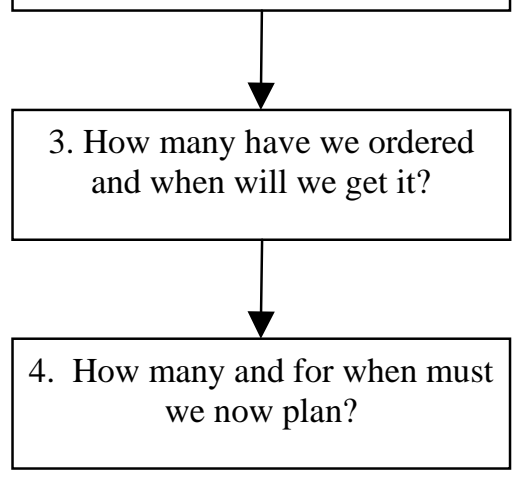

Figure 5: Orlicky's algorithm [6] 
four-step algorithm involve only calculations and reading from a database; these can be easily programmed and executed sequentially in a single batch run and many computer systems were developed to do just that. These systems became known as "Material Requirements Planning” or MRP systems.

When time-phasing is added in, MRP would in Question 4 determine whether any material on order will arrive in time to satisfy the demand determined in Question 1 or not. Should the answer be "no", the computer system generates an action message and essentially hands the problem over to a human planner to find a solution.

Should the quantity in stock and on order be insufficient to satisfy the demand, as in the numeric example above, the computer recommends that a supply be scheduled using fixed order policies and fixed lead times stored in its database as static data. Should the actual lead time or batch quantity vary, the human planner is expected to override the computer's plan "to adjust for reality”.

In terms of the flow diagram of figure 2, reproduced here as figure 6, one can summarise the Requirements Planning methodology by saying it consists of two parts:

1. The MRP computer system which develops a plan that is likely to be feasible by using static assumptions, and

2. The team of human planners that is responsible for ensuring that a feasible plan is indeed produced.

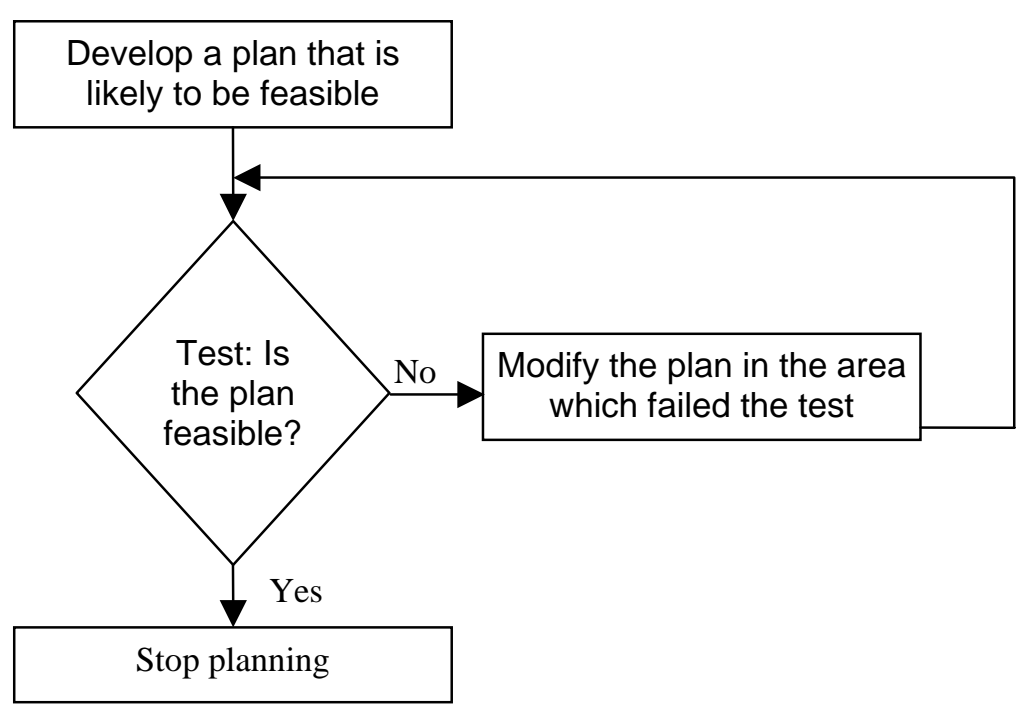

Figure 6: Developing a feasible plan

At no point does Requirements Planning, either in Orlicky's original formulation of the algorithm or in the MRP computer system, consider alternative plans; the algorithm just sequentially processes the four-question algorithm, reads the static assumptions and delivers the result as "the” plan.

\section{MRP II AND ERP}

Although, MRP in the computer system evaluates neither feasibility nor optimisation when creating the supply plan, Orlicky's algorithm can be shown to create a material plan that is inherently superior to the most common alternative, order point planning. With good human planners to ensure feasibility, manufacturing companies in the seventies could and did achieve very significant business benefits by replacing order point planning, planning boards and manual methods with MRP systems. 
Over the next three decades, computer systems based on Requirements Planning expanded to include capacity planning and distribution requirements planning (using similar algorithms) and to integrate the execution control processes of purchasing, inventory control, production control and sales control. Applications also expanded beyond the manufacturing industry. A whole body of knowledge developed around what became known as Manufacturing Resource Planning (MRP II) and later Enterprise Resource Planning (ERP). The flow chart of figure 7 describes MRPII as defined by one of the important protagonists of the time, Oliver Wight [14 and 15].

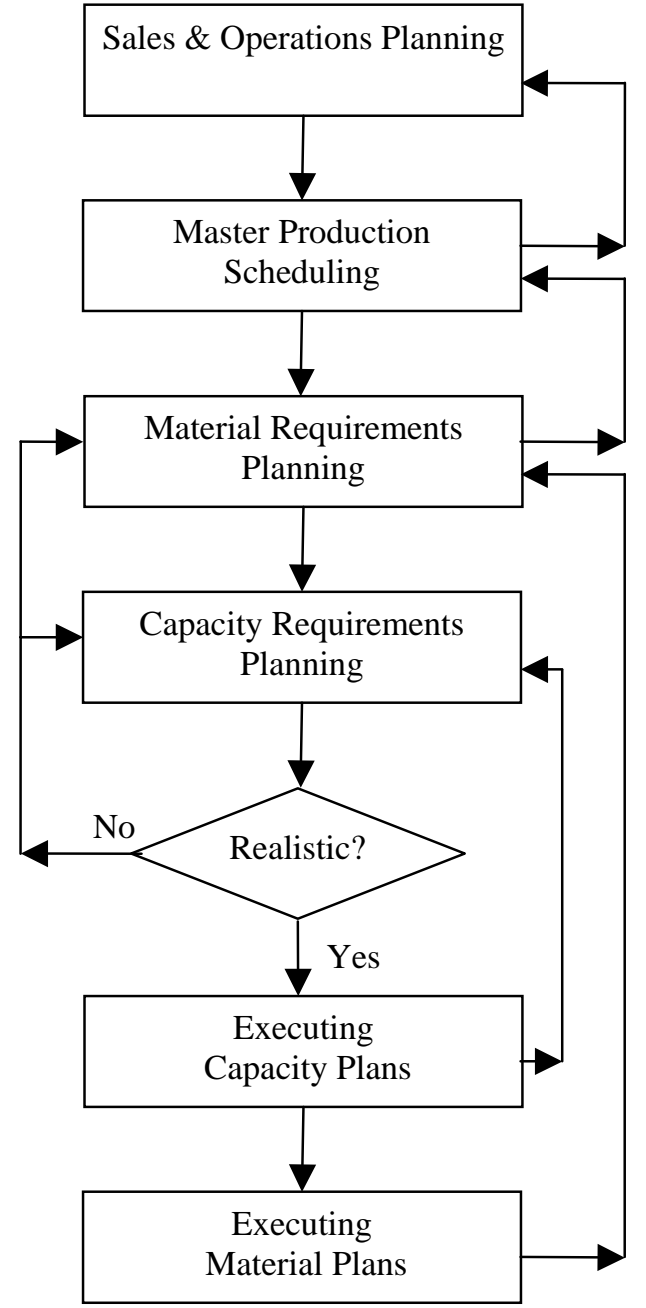

Figure 7: MRPII [14]
As far as planning processes go, the MRPII and ERP body of knowledge focuses on synchronising aggregate level plans (sales \& operations plans) with product level plans (master schedule and distribution requirements planning) and subsequently with material and capacity plans (material requirements planning, capacity requirements planning and roughcut capacity planning). The computer system sequentially calculates each level to ensure that the plans at different levels are synchronised; the planners and schedulers have to ensure that each level's plan is feasible. If a plan is not feasible, the master scheduler or the planner has to make adjustments to ensure that the problem is corrected.

Significantly, in Oliver Wight's [14] diagram in figure 7, the flow diagram checkpoint "Realistic?" as well as all of the feedback loops are performed by human planners. A good summary of MRP II is that the computer performs all the top down planning; the humans do all the bottom up feed back.

The MRP II systems of the late seventies and the eighties evolved into the ERP systems of the nineties, almost all of it by expanding the scope of transactionbased business processes covered by the system and building multi-site and real-time functionality. The planning processes of ERP systems are essentially the same as those of MRPII systems in terms of methodology.

These MRPII / ERP planning processes put the emphasis on developing feasible plans (ensured by human planners) that are synchronised (by the computer system). The synchronisation from executive level long range aggregate plans down to detail short range material and capacity plans goes a long way to deliver superior plans without requiring the rigorous certainty that the best possible plan is selected. For a long time this has been "good enough for practical purposes" and it is likely to remain a reasonable way to plan real businesses for many years to come. 


\section{SYSTEM DEVELOPMENT}

In the 1980's and the 1990's computer capabilities accelerated to such an extent that new methods that depend solely on computer power were developed to solve old problems.

One significant development involved using algorithms executed in a computer system to generate alternative solutions for a particular problem and to check each solution for feasibility. Some of these would end the planning process when a feasible solution is found while others would continue to generate alternative feasible solutions.

The latter systems typically use rule-based logic or heuristic methods which include an objective function in order to select the "current best" plan from amongst the feasible alternatives found so far and to direct the search for even "better" solutions. Some of these algorithms terminate the search when a pre-defined threshold is reached (for example Shobrys' [8]) "give me the best solution you can find in $x$ time") and may therefore never find the true optimum.

Consider again the requirements for optimisation listed in section 4 "A Comparison of Methods” above:

1. Consideration of alternative plans.

2. Selection of the feasible, optimum plan.

3. The knowledge that no better plan exists.

Heuristic and rule-based methods do not represent "true" optimisation in the sense that they do not satisfy the third requirement, the knowledge that no better plan than the current best solution exists. The distinction is important; however early adopters, especially in process manufacturing such as chemicals and oil, established that just finding a near-optimum or even a more limited form of optimisation as represented by heuristic methods or rule-based algorithms deliver real business benefits. In practice one nowadays often finds that these methods are also referred to as "optimisation".

For the purpose of this article we will accept this "less pure" terminology and refer to optimisation as including any method that considers a number of alternatives (not necessarily all), validates them for feasibility and selects the best in terms of an objective function.

As computer systems evolved over the last two decades, though, "true" optimisation systems were also developed, solving optimisation problems with quantitative techniques and highend computer power and inherently satisfying the third requirement above. Originally only fairly simple problems could be modelled using mostly linear programming but progressively the scope and complexity of problems that could be optimised expanded.

\section{SUPPLY CHAIN PLANNING}

A significant development in the commercialisation of the systems described in the previous section was their application to supply chain planning for manufacturing and distribution companies. 


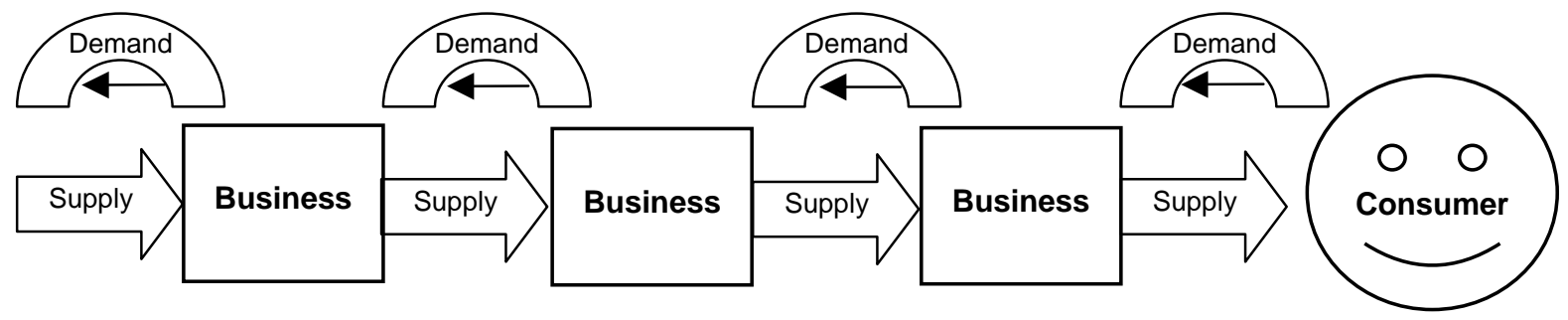

Figure 8: A Supply Chain

A supply chain such as that depicted in figure 8 typically links numerous business entities in such a way that "upstream" businesses satisfy demands from "downstream" business all the way down the supply chain to satisfaction of the independent demand from the end consumer. Supply chain planning involves the development of supply plans to satisfy the demands at every step. Industry have found that significant business benefits in lower inventory levels, increased customer service and lower logistics cost can be realised by improved supply chain planning. Due to the complexity and the volumes of data, though, optimising supply chain planning, that is creating the best possible supply plan to meet the demand, very rapidly becomes a challenging exercise for even reasonably straight forward supply chains. Systems that can support this planning process thus have immediate and real business value.

Solving supply chain optimisation problems with computer systems became increasingly practical and profitable, especially over the last decade, for both the companies selling the technology and the businesses making use of the technology. Specifically for developing optimum supply plans to meet a demand, practical ways were developed to separate the "building" of the optimisation process from the "using" of the process to create an optimum supply plan.

Generic optimisation using linear programming or similar



Optimising supply chain planning using computer systems

Once:

- Define the objective function

- Define the nature of the demand

- $\quad$ Define the constraints

Every time re-planning is required:

- Get the values of the constraints

- Get the values for the demand

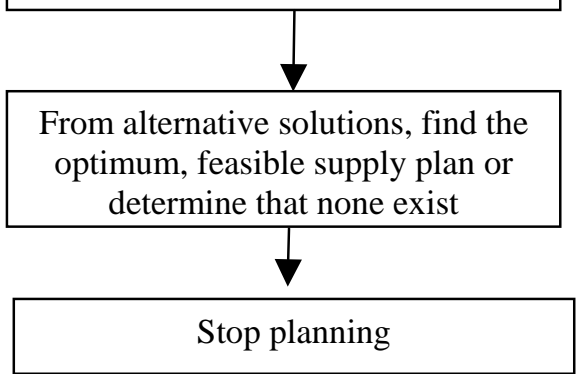

Figure 9: Optimisation for supply chain planning using computer systems 
With reference to the four steps of the optimisation process using linear programming as described in figure 4, it was found in practice that the objective function in supply chain planning is unlikely to change every time re-planning is required and so it can be set-up once only. Similarly, the constraints that determine a feasible supply chain planning solution are likely to remain the same although the actual value of the constraints may vary. (In the scheduling example above, the time available in the work-shift remains the constraint but the actual length of a work-shift may change.)

On the other hand, every time there is a significant change in the value of the demand or in the value of the constraints determining the ability to supply to the demand, re-planning is required. The net effect is that supply chain planning where a computer system is used to consider alternative plans, evaluate them for feasibility and produce some form of optimised supply plan, effectively restates the flow diagram of figure 4 , repeated on the left side of figure 9 , into the form of the right side of figure 9.

\section{ADVANCED PLANNING}

Optimising supply chain planning in the manner of the right side of figure 9 is Advanced Planning. The essential components, presented graphically in figure 10, are the following:

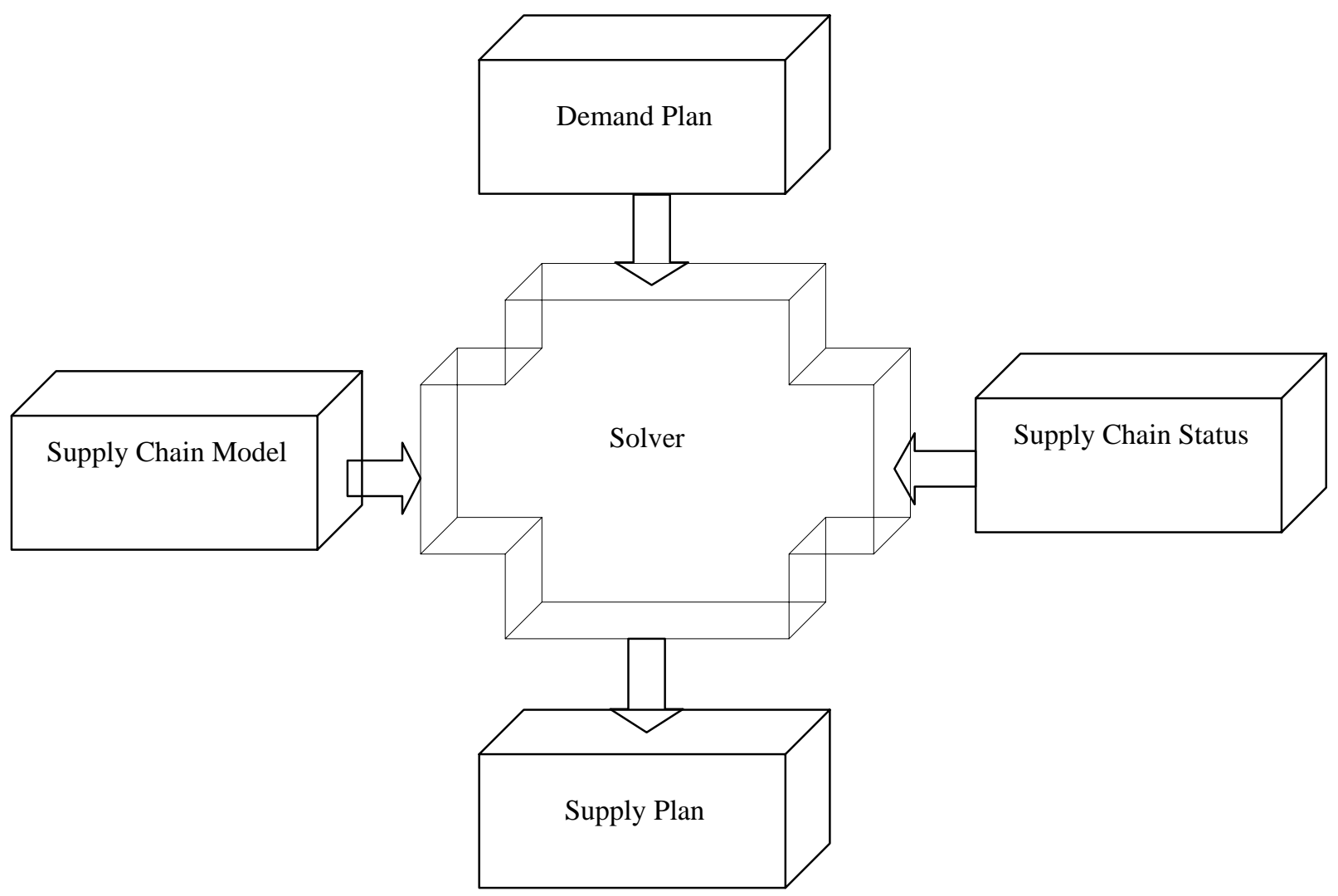

Figure 10: Advanced Planning 
- The "Supply Chain Model" describes the objective function, the nature of the demand and the constraints.

- The "Demand Plan" quantifies the current values for the demand.

- The "Supply Chain Status" quantifies the current values for the constraints.

- The "Solver" finds alternative plans, tests for feasibility and selects the optimum.

- The "Supply Plan" represents the best way to satisfy the current demand, given the current status of the supply chain.

\section{SUPPLY CHAIN MODEL}

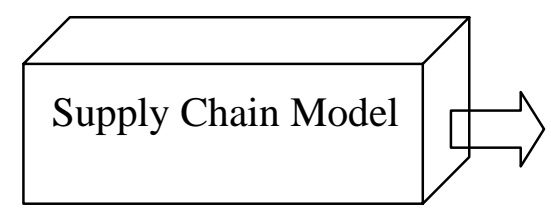

Figure 11: Supply Chain

The supply chain model describes the specific aspect of the supply chain that is being optimised. This design activity is performed once during the "build" phase to set up an Advanced Planning business process. It may of course be modified as time goes by but is considered to be static data in the planning process.

Stadtler \& Kilger [9] in 2000 classified the many different aspects of a supply chain that one may wish to optimise - and therefore the different supply chain models that may be constructed - into three basic groups derived from the planning horizon of the optimisation problem:

Long term supply chain planning is often called "strategic planning" and is usually applied to problems involving the optimisation of the design of the supply chain over multiple years. A typical long range supply chain model would be one constructed for a business with multiple facilities in order to determine the optimum geographic locations for these facilities (for example manufacturing plants and/or distribution centres) and the allocation of market segments that each should serve. The objective function is usually stated as maximising profit or minimising cost.

Medium term supply chain planning puts the focus on the demand and supply for individual products along the supply chain. A typical Advanced Planning application would be to determine the optimum supply plan for a specific demand where the supplying business could produce the product in any of a number of different factories and deliver it via a number of different delivery channels. Medium range supply chain models usually optimise over many months with a planning horizon closely aligned with a financial year or the cumulative lead time of the products.

Short term supply chain planning makes use of Advanced Planning to solve scheduling problems, for example to determine the optimum sequence for manufacturing or distribution activities. The example used earlier in this article, sequencing products on a machine to minimise total manufacturing time, is an example of a short term supply chain planning problem.

\section{SUPPLY CHAIN STATUS}

The supply chain status quantifies the current values of the constraints and other parameters and variables that are used in the supply chain model. 


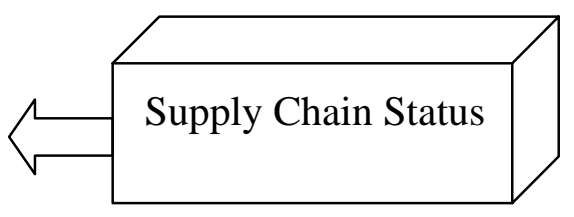

Figure 12: Supply Chain Status

In the short term optimisation (scheduling) problem described earlier in this article, for example, the length of the work-shift is a constraint because the planner's schedule will only be valid if all the products can be processed within the work-shift. Now it may be that the length of a work-shift differs from time to time; Friday afternoon shifts may be shorter and Saturday morning shifts may be longer and there may be special times of the month or the year when the shifts are different from usual. Every time the planner uses Advanced Planning to develop an optimum plan, he or she must provide as an input the length of the particular shift for which the schedule is being developed.

In supply chain optimisation problems the quantitative data required by the supply chain model are supply chain status information such as current inventory levels, available capacity, open orders and so forth - information nowadays typically held in the database of the Enterprise Resource Planning (ERP) system that most supply chain businesses use to manage transactions in purchasing, production, inventory control, sales and finance. The implementation of Advanced Planning at this time (end of 2003) for this reason usually require significant software engineering to integrate the databases of the Advanced Planning system and the ERP system.

The expectation is that increased use of templates for supply chain model development and increased standardisation of ERP system interfaces will streamline this (currently) very costly and time consuming aspect of Advanced Planning implementation.

\section{DEMAND PLAN}

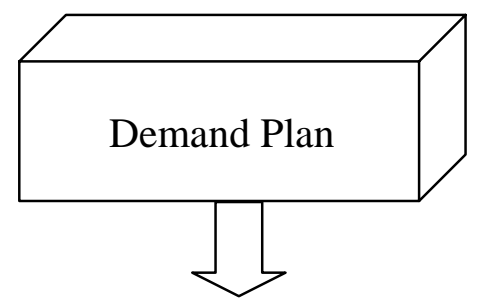

Figure 13: Demand Plan

Advanced Planning was defined earlier in terms of finding an optimum supply to meet the demand. This demand is usually a mixture of forecast demand, actual orders, long term contracts, call-off schedules and other demand types that change from one status to the other over time. In addition, each of these demand types is often stated in different configurations such as by product family, by product, by customer, by customer industry, by customer geographic location, and so on.

This complexity is usually managed by devoting a significant portion of the capabilities built into the Advanced Planning software to demand management. This often includes forecasting functionality that compares with or exceeds the capabilities of stand-alone forecasting systems.

In fact, the ability of Advanced Planning systems to comprehensively and comprehensibly manage demand have motivated many businesses to buy and implement only the demand management module of a particular Advanced Planning system to great benefit. It is also a typical starting point for implementation projects that elect to follow a step-by-step implementation of a high-end Advanced Planning system. 


\section{SOLVER}

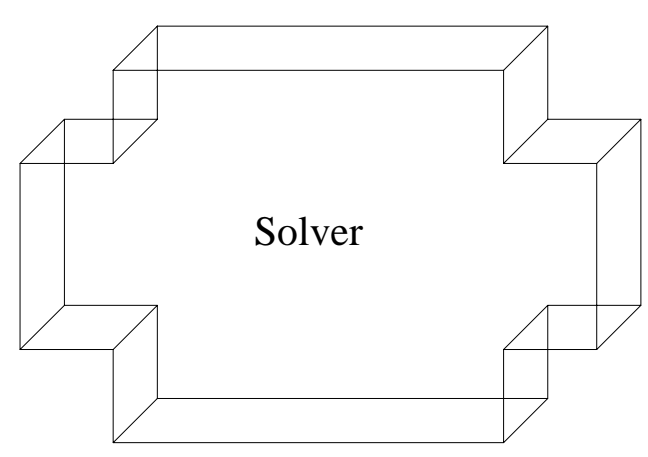

Figure 14: Solver

The Solver is the part of the computer system that finds the optimum supply to meet the current demand given the current status of the supply chain in terms of the specified supply chain model. (The term "optimiser" is sometimes used.) It does this by ensuring that only feasible alternatives are considered and does it "in one go" as opposed to for example the sequential steps in the MRPII / ERP planning processes discussed earlier.

Businesses may elect to construct their own supply chain models, build the integration paths to the ERP system to obtain the supply chain status data and use the ERP system or stand-alone systems to provide input. Almost nobody, however, elects to write their own Solver so even businesses building their own Advanced Planning in this manner will buy a commercial Solver.

Early Solvers were essentially linear programming algorithms or rule-based expert systems. Nowadays, the exact algorithms used are often considered trade secrets and protected by copyright.

\section{SUPPLY PLAN}

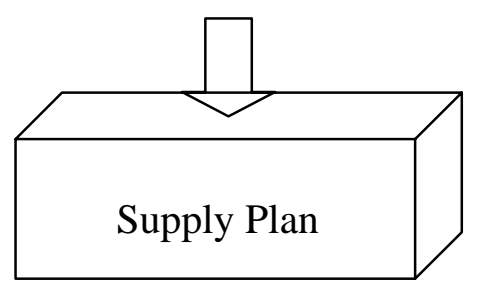

Figure 15: Supply Plan

The supply plan is the Advanced Planning output, as defined in the supply chain model.

In the section above on Requirements Planning the point was made that in MRPII / ERP planning the computer system creates a synchronised plan - and only that. It is then up to the planners to ensure that the plan is feasible. Although the computer system provides significant support, the process is incomplete without the human actions.

Advanced Planning on the other hand, delivers the final supply plan as a computer output, having already considered various alternatives, verified feasibility and selected the optimum.

This strength of Advanced Planning is also a weakness in that in practice one often encounters serious misgivings to the unquestioning execution of a plan created by a computer. If sufficient resistance occurs, execution will be only partial or fragmented, leading to suboptimum results which leads directly to loss of credibility and from there to failure of the planning process.

As a minimum, practical experience indicate the value of having appropriately authorised and accountable personnel confirm the supply plan before execution. 


\section{COMMERCIAL SYSTEMS}

As indicated in the section on the Solver above, organisations sometimes construct their own Advanced Planning applications by developing a supply chain model, integrating this with their ERP system and buying a commercial Solver.

Much more common, though, are the commercial systems where a single vendor provide an integrated solution with supply chain modelling tools, demand management and supply chain status capability and either an in-house developed or a third-party Solver. Such a computer system using Advanced Planning as its planning methodology is referred to as an Advanced Planning System or APS. (The term “Advanced Planning Solution” is also used.)

The range of supply chain models covered by a particular Advanced Planning System (discussed in section 11 above from the work by Stadtler \& Kilger [9]) is a useful primary classification approach. The current status (at the end of 2003 in a field that is evolving rapidly) is that there are only a handful of "leaders" who provide the full scope from long term strategic planning through medium term product level planning to short term scheduling and sequencing. There are, however, a very large number of Advanced Planning Systems that offer so-called "point" solutions; addressing a particular supply chain planning problem and therefore pre-packaged with a template to rapidly construct a particular supply chain model. This is especially true for short term scheduling and sequencing of some specific aspect of the supply chain.

Typically the leaders deliver stand-alone systems for long term (strategic) planning and separate modules in a single system for medium and short term planning. The system also typically has a robust demand management system that can replace demand management in the ERP system or any stand-alone system that may exist. Furthermore the APS would have integration tools to extract the supply chain status from a number of alternative ERP systems, especially from an ERP system sold by the same owner. (Some ERP vendors now provide an APS as part of their bundled offering.)

The computer technology used by the leaders is sometimes so advanced that the APS system provides capabilities outside the scope of basic Advanced Planning but similar and sometimes better than the more traditional ERP systems, Customer Relationship Management (CRM) systems or Business Intelligence (BI) systems. This technology also allows the leading Advanced Planning Systems to support multi-site planning in a way that ERP in practice didn't quite achieve even though multi-site is considered an inherent part of the move from MRPII to ERP. (In our opinion, one of the reasons for this failure of ERP to live up to its promise is that human planners find the multi-site environment too complex to manage using the limited computer tools available to them under the MRPII / ERP methodology.)

There are now commercial systems on the market that started out as "pure" APS but have added such extensive functionality in supply chain management and data management that the point is sometimes reached where the existence of a separate, stand-alone ERP system is not required.

With reference to the discussion in section 8 "System Development" above, a secondary way to classify an APS (after the scope of the supply chain modelling functionality) is to 
determine whether it delivers a "true" optimum or merely a rule-based or heuristic "current best" solution from those evaluated. There is some difficulty, though, in comparing the quality of the optimisation delivered by different systems. For example, Goldratt [5] devotes a significant part of his book "Necessary but not Sufficient" to the question of how one can validate a claim of "true optimisation" where the Solver algorithms are trade secrets.

\section{APPLICATION}

When considering the use of Advanced Planning, the key issues - of course - involve the business benefits achievable and the cost and risks of using Advanced Planning instead of one of the alternative methodologies to solve a particular supply chain planning problem. The discussion in this section follow the long term, medium term and short term planning classification of Stadtler \& Kilger [9].

Solving a long term strategic supply chain planning problem is an infrequent exercise for most businesses and so the distinction between the "build" and the "use" of the planning process tends to blur. The leading APS suppliers package their long term strategic planning solutions as stand-alone systems usually not integrated with the ERP system or other modules in the APS.

The once-off nature of these problems and the fact that there is (usually) weeks or months available to come up with a solution, make it reasonable to construct a custom built planning process. If the problem-complexity is manageable, manual solutions fortified with spreadsheets are common. If the problem is complex, many businesses use linear programming, simulation and other operations research techniques. A Strategic APS can be seen as an alternative to the latter and reported case studies are on the increase.

A strategic planning problem typically involves large investment decisions (build a warehouse, close a factory and so on) and it is therefore worthwhile spending reasonable time and money to develop a good solution. The risks in the planning process, however, do not lie in the tools used but in the accuracy of the data and the long range assumptions one is forced to make. Advanced Planning has proven useful for long term supply chain planning but so have simulation, linear programming and other approaches.

For medium term supply chain planning MRPII / ERP is at this time (the end of 2003) by far the most commonly used methodology. There exists a whole body of knowledge, years of experience by many people all over the world and consensus on the best practices to be followed when using this particular methodology to plan production and distribution, synchronise dependent material and capacity plans and use these plans to drive purchasing, production, distribution and sales activities.

On the other hand, there can be little argument that from a purely theoretical point of view Advanced Planning delivers better supply chain planning solutions than the Requirements Planning methodology of MRPII /ERP - in fact its delivery of the best solution is inherent in the Advanced Planning methodology.

Outside this conceptual viewpoint, though, Advanced Planning suffers from a number of drawbacks when implemented in actual supply chains: 
- The supply plan produced by the computer system cannot practically be verified by human analysis and must therefore be executed on the assumption that "the computer knows best”, a very, very questionable proposition in many business situations.

- Advanced Planning is even more reliant on data accuracy than Requirements Planning. Any degrading in the accuracy of the input data, especially the supply chain status, will rapidly lead to obviously sub-optimal supply plans being produced. This leads to loss of credibility with consequent reluctance by human personnel to blindly execute the supply plan. A supply plan which is NOT executed, for whatever reason, is of course literally useless - which means the failure of the whole Advanced Planning business process.

- At this time (end of 2003), an APS with medium term supply chain planning capability is expensive to purchase, the implementation is costly and time-consuming and highly paid personnel are required to use it. Advanced Planning is clearly the high-cost option.

- At this time (end of 2003), best practices and experienced personnel who know how to use Advanced Planning are in short supply.

Generally speaking, the advantages of using Advanced Planning increase with increasing complexity of the supply chain planning environment. In simple planning environments, the business benefits of having an optimum supply plan, as delivered byAdvanced Planning, may be marginal compared with the "good enough for practical purposes" supply plan delivered by Requirements Planning or even visual or other manual techniques. When the disadvantages listed above are added in, Advanced Planning in this environment is sometimes deemed not worth the cost and risk.

Short term supply chain planning covers a range of scheduling and sequencing problems that MRPII and ERP systems only address by planning start and end dates - literally; the smallest time increment of the Requirements Planning methodology is one day.

Scheduling and sequencing in industries such as oil, steel and chemicals require by the hour or even finer schedules. In addition, feasibility in these industries is usually quantified by "hard" constraints (for example there is no practical, short term way to adjust the capacity of a container). Many of the "point solutions" referred to in the section above were initially developed "in the field" by businesses in similar circumstances building there own Advanced Planning Systems and never following the Requirements Planning route of the last quarter of the twentieth century.

Generally speaking, the costs and risks, although similar, are lower when using Advanced Planning for short term scheduling and sequencing than the medium term planning situations discussed above. In some businesses there are significant business benefits to be gained by following a superior schedule; in others the benefits are marginal. As with all projects involving computer systems, one should therefore first establish a clear view of the supply chain optimisation problem that needs solving, quantify the business benefits, the costs and the risk, and then decide whether to use an APS or an alternative methodology.

In general, when weighing the applicability of Advanced Planning against the alternatives, a key point to bear in mind is that most businesses today (end of 2003) already have an ERP system. The purveyors of Advanced Planning Systems often dismiss ERP as a transaction system only but this is erroneous. The Requirements Planning methodology embedded in ERP can and is used by many businesses, sometimes exclusively and sometimes in 
conjunction with other planning methodologies, including Advanced Planning.

Nevertheless in practice it is common to find that businesses use only the transaction processing capabilities of their ERP system. Before embarking on Advanced Planning to solve all of the planning needs of a business, an obvious question to ask is whether Requirements Planning is not used because it is inappropriate and whether using Advanced Planning will resolve the issue.

A non-trivial question to ask is whether a business has the organisational capability required for optimisation. For instance, the example of medium term supply chain planning mentioned earlier described how an APS, probably run by a centralised corporate planning department, would develop the plans determining which products must be manufactured when in each of multiple plants in order to optimise corporate profit or cost goals. Implementing such a system in an environment running, say, stand-alone MRPII systems up to now, effectively means removing accountability for all production planning from the plant manager; instead he or she must henceforth "just do what the plan coming out of the APS system at Head Office tells you to do".

\section{SUMMARY}

Far from seeing Advanced Planning as some form of "the next version of planning beyond Requirements Planning”, practitioners should take from this article the key principle that Advanced Planning is based on a different methodology from that of Requirements Planning or other alternatives. The question should really be: "Which methodology should be used to solve our particular supply chain problem?”

Visual and other manual planning methodologies do not use computer systems at all. With Requirements Planning the computer system creates a single synchronised plan that is likely (but not assured) to be close to feasibility. Since there is only one plan there is no question of optimisation.

The Advanced Planning methodology relies on the computer to find alternative feasible supply plans to meet the current demand plan given the current status of the supply chain constraints and to select the optimum, all in a single step without human interaction.

Any business decision to use Advanced Planning instead of an alternative methodology should take into consideration the likelihood of business benefits, the cost and the risk. Thinking around the risks should specifically consider the credibility of computer generated supply plans and the disempowerment of local decision makers in order to achieve a global optimum.

Over time, Advanced Planning will gain its own body of knowledge, its own consensus best practices and large numbers of protagonists with significant practical experience in many different supply chain planning situations. This should also lead to more acceptance of "the plan produced by the computer" and the new and different roles and responsibilities required when using this methodology. If in addition the cost of buying and implementing an Advanced Planning System becomes more manageable, the adoption of this methodology is likely to accelerate. 


\section{REFERENCES}

[1] Chase, R.B., Aquilano, N.J. \& Jacobs F.R., "Production and Operations Management: Manufacturing and Services”, Eight Edition

[2] Goldratt, Eli M. (with Jeff Cox), 1984. “The Goal”, Creative Output, Netherlands, 262 pages.

[3] Goldratt, Eli M. (with Robert E. Cox), 1986. “The Race”, North River Press, New York, 179 pages.

[4] Goldratt, Eli M., 1990. “Theory of Constraints”, North River Press, New York, 162 pages.

[5] Goldratt, Eli M. (with Eli Schragenheim and Carol A Ptak), 2000. "Necessary but not Sufficient”, North River Press, Massachusetts, 231 pages.

[6] Orlicky, Joseph, 1967. "Material Requirements Planning”, New York et al.

[7] Pienaar, Abré, 1991. "Vervaardigingstelsels in die Praktyk", Nobel Uitgewers, Centurion, 169 pages.

[8] Shobrys, Donald E, 2001. “The history of APS”, Supply Chain Consultants, www.supplychain.com, 8 pages.

[9] Stadtler, H \& Kilger, C, 2000. "Supply Chain Management and Advanced Planning, Concepts, Models, Software and Case Studies”, Springer, Berlin, 371 pages.

[10] Supply Chain Council, Inc, 2002. "Supply-Chain Operations Reference-model, Overview of SCOR Version 5.0”, www.supply-chain.org.

[11] b, 2003. Unpublished memoranda, discussions and workshops.

[12] Vollmann, T.E., Berry, W.L. \& Whybark, D.C. "Manufacturing Planning and Control Systems".

[13] Winston, Wayne L, 1994. “Operations Research: Applications \& Algorithms”, Third Edition, ITP, London.

[14] Wight, Oliver W, 1982. “The Executives Guide to successful MRP II”, Oliver Wight Limited Publications, Vermont, 104 pages.

[15] Wight, Oliver W, 1982. “MRP II: Unlocking America’s Productivity Potential”, Oliver Wight Limited Publications, Vermont, 555 pages. 